\title{
Culture vs NAATs: Is the playing field level in diagnosis of gonorrhoea?
}

\author{
David Barlow MA BM BCh FRCP FRCP (Edin) FSLCoSHH \\ Emeritus Consultant Physician, Department of Sexual Health (GUM), Guy's and St Thomas' NHS Foundation Trust, \\ Fellow of Sri Lanka College of Sexual Health and HIV Medicine, Emsil: Email: davidbarlow@doctors.org.uk, \\ (iD https://orcid.org/0000-0001-6997-1292.
}

In 2017 the journal Sexually Transmitted Diseases (STI) published a series of 300 word vignettes celebrating the centenary of genitourinary medicine services in the UK. Each invited piece was to be based on two articles published in STI or its predecessors (Genitourinary Medicine, and the British Journal of Venereal Diseases). I chose to feature gonococcal culture ${ }^{1}$ and referenced two of our speciality's great names, Harrison ${ }^{2}$ and Catterall ${ }^{3}$. I was asked to provide a longer 'blog' on the topic but, as this contribution is only available to the Journal's online subscribers, there is no printed version. The piece that follows is an expanded argument based on, and includes a little material from, the STI contribution and revisits a topic to which I have returned over the years at The College's Annual Academic Sessions ${ }^{4-7}$.

There were significant improvements in laboratory culture of gonorrhoea during the 1970s but it was 30 years before revolutionary techniques for diagnosis became commercially available. These alternative tests, Nucleic Acid Amplification Tests (NAATs), were simpler to administer, but their introduction brought loss of personnel and diminished need for microscopy and laboratory skills. They also introduced a 'commercial imperative' makers of diagnostic tests need to recoup their development costs and produce profit for themselves and their shareholders. For this they have to show superiority over laboratory culture.

I highlighted this potential conflict of interest in my 2012 talk $^{6}$ with an allegory of a carrot farmer presented with a new commercial test for identifying his produce. While this test was claimed to be superior to the traditional 'see, touch, taste method', it was expensive and from time to time identified a turnip as a carrot.

Laboratory culture of Neisseria gonorrhoeae lacks, and has always lacked, $100 \%$ sensitivity. In the pre-NAAT era, samples were taken from multiple sites, on multiple occasions, to diagnose, to exclude, and, importantly, to monitor any fluctuations, in the efficiency of laboratory culture. Such use of repeated tests to monitor the sensitivity of culture is now impossible following the universal adoption of treatment of contacts (epidemiological) before diagnosis.

In the 1970s, tests were taken on at least three separate occasions to diagnose or exclude gonorrhoea in women. Even with multiple sets of tests, the culture results varied significantly between Centres.

The decade was marked by attempts at improving cultural sensitivity: a variety of selective but non-inhibitory mediums, usually modifications of Thayer-Martin's version, gradually replaced non-selective ones. Catterall however used McLeod's culture medium, with no antibiotics or antifungal agents, and only diagnosed $60 \%$ of gonorrhoea at the first visit.

A series of publications from London clinics ${ }^{8}$ demonstrated laboratory culture's lack of sensitivity but its gradual improvement. Results from the first set of tests at various Centres included 66\% (Middlesex, 1970²), 90\% (St Thomas', $1971^{9}$ ), 91\% (Middlesex, $1976^{10}$ ), 88\% (Charing X, 1976 ${ }^{11}$ ), 97\% (St Thomas', $1976^{12}$ ), 98\% (St Thomas', 1978 ${ }^{13}$ ), 95\% (Barts, $\left.1979^{14}\right)$. All of these figures came from units 
with a particular interest in gonorrhoea and its diagnosis, but variation persisted ${ }^{1}$.

Duncan Catterall's 1970 article demonstrates sharp contrasts with today's practice and prevalence* of infection:

"Every patient had at least 4 pelvic examinations and the majority had 6 or more genital tests. ${ }^{3}$ "

Exclusion was as important as diagnosis. We knew that the first set of tests would miss some cases but we also knew that between $8 \%$ and $35 \%{ }^{15}$ of gonorrhoea contacts would not have the disease, (which polarised views for and against epidemiological treatment). The same may be true today: Turner writes ${ }^{16}$ "Presumptive or epidemiological treatment of chlamydia and/or gonorrhoea accounts for a large number of suboptimal and unnecessary antibiotic prescriptions", calculating (" $a$ conservative assumption") the probability of partner infection as 0.4 .

"Gonococci were found [by microscopy] in only 67 (69\%) of 95 consecutive cases ${ }^{3 \text { " }}$

This last quotation demonstrates how 'a poor culture service flatters the microscopist ${ }^{17}$ ': Suppose 50 of 100 genuine cases of gonorrhoea $(50 \%)$ are identified by microscopy. If culture misses 20, microscopy will appear more sensitive, at 50 of 80 (62.5\%). The same calculation applies to assessment of NAATs.

Microscopy in women at St Thomas's found $50 \%$ (implying better culture results) compared with Catterall's 69\%, and a later retrospective survey ${ }^{18}$, from Catterall's unit, noted the poorer sensitivity of culture in small clinics outside London. Again variation.

Use of Ian Phillips' VCNT ${ }^{9}$ combined with scrupulous attention to detail, had improved culture sensitivity to $98 \%$ by 1978 (continuing at $97 \%$ in $1988^{19}$ ).

Which brings me to the big question: how does the standard and variability of gonococcal culture today compare with (the best of) the 1970s? And does it matter?
In 1977 Morton $^{20}$ wrote "The gonococcus is the most fastidious of organisms. It has long taxed the skill and ingenuity of bacteriologists." The WHO, with Unemo and Ison's imprimatur $(2013)^{21}$, updates the caution: "Strict sample collection, transportation, and storage are crucial to maintaining viability", listing vital criteria: "Number of sampling sites, technique and swabs used for collection of specimens; conditions and duration of transportation; composition and quality of the culture medium; inoculation and incubation conditions; and reagents and techniques used for the species identification of $N$. gonorrhoeae."

In the 1970s, as now, we had Reference Laboratories, guidelines, and exhortations to quality control and yet there was still a measurable variation in outcomes of gonococcal culture between and within London's 'Centres of Excellence'.

Before I am overwhelmed by howls of protest from microbiologists (and epidemiologists), I wonder how many laboratories, whose 'culture' results provide today's comparators for epidemiological and NAAT evaluations, look, have looked, at the performance of their transport/culture systems ${ }^{22,}{ }^{23}$, in a similarly fastidious way?

What proportion of clinics or testing sites these days have all the index samples plated direct in clinic on to an appropriate selective but non-inhibitory medium (made up inhouse as required, not commercial $\left.{ }^{23}\right)$, and placed directly in the clinic's $\mathrm{CO}_{2} /$ humidity/ temperature-controlled incubator, with samples transferred to the on-site laboratory (dedicated gonorrhoea bench, dedicated gonorrhoea technician) twice daily, and with daily quality control to include correlation of microscopy of samples from the male urethra with their culture results.

This crucial monitoring of men's samples enabled us to pick up problems with the incubators (clinic and laboratory), growth medium, a new technician on the ' $G C$ ' bench, 
or even sloppy plating-out in the clinic. If a discrepancy arose, we were aware of it in as few as 24 hours and were able to adjust our management of women, for whom culture was (and should still be) of so much greater importance.

While matching the earlier paragraph's stringent conditions, culture in 1988 at St Thomas' still missed $3 \%$ of cases of gonorrhoea in women at the first attempt ${ }^{19}$.

Nobody would disagree with the conclusion of a recent STI article ${ }^{24}$ that: "...the use of dual NAATs in the context of a population with low prevalence of gonorrhoea is likely to result in false positive results", but one should also question any who assume that all the NAAT positive/culture negative results in their series are false positives.

I end with two cautions ${ }^{1}$; one old, one new:

Taylor and Phillips (1980): "We conclude that although these transport-culture media performed well if incubated immediately and examined immediately, performance was not nearly so good under conditions that mimic more closely those actually obtaining in most clinics." ${ }^{23}$

And my own (2017): ' $a$ poor culture service flatters the NAAT, and may distort measures of prevalence and incidence'.

* Catterall's 1970 paper described a consecutive series of 300 women who had attended his Central London clinic because of vaginal discharge. They included 95 cases of gonorrhoea. A similar series in the UK today would be unlikely to include ${ }^{9}$.

\section{References}

1. Barlow D (2017) Culture of the gonococcus: a reliable gold standard? Sex Transm Infect; 93, 3, 178. http://dx.doi.org/10.1136/sextrans-2016-053004

2. Harrison LW (Colonel) (1927) Gonorrhoea. Br.J.Vener.Dis. 3, 24-32

3. Catterall RD (1970) Diagnosis of vaginal discharge. Br.J.Vener.Dis. 46, 122-4
4. Barlow D (2006) Diagnosis of gonorrhoea - are we going backwards? 11th Annual

Academic Sessions, College of Venereologists of Sri Lanka, Colombo

5. Barlow D (2008) Problems with Diagnostic tests. $13^{\text {th }}$ Annual Academic Sessions,

Sri Lankan College of Venereologists, Colombo

6. Barlow D (2010). PCR vs. Culture in the diagnosis of GC and Chlamydia; way forward

$15^{\text {th }}$ Annual Academic Sessions, Sri Lankan College of Venereologists, Colombo

https://www.researchgate.net/publication/314096628_ PCR_vs_Culture_with_2017_addendum

7. Barlow D (2012) Lies, damned lies and STItistics $17^{\text {th }}$ Annual Academic Sessions Sri Lankan College of Venereologists, Colombo

8. Barlow D (2007). R.D. Morton Memorial Lecture, BASSH Spring Meeting, Blackpool

https://www.researchgate.net/publication/270567127_ Gonorrhoea_(slide 30)

9. Thin RN, Williams IA, Nicol CS (1971). Direct and delayed methods of immunofluorescent diagnosis of gonorrhoea in women. Br.J.Vener.Dis. 47, 27-30

10. Chipperfield EJ, Catterall RD (1976). Reappraisal of Gram-staining and cultural techniques for the diagnosis of gonorrhoea in women. Br.J.Vener.Dis. 52, 36-39

11. Evans BA (1976). Detection of gonorrhoea in women. Br.J.Vener.Dis. 52, 40-42

12. David Barlow, Nayyar K, Phillips I and Barrow J (1976). Diagnosis of gonorrhoea in women. Br.J.Vener.Dis. 52, 326-328

13. David Barlow and Ian Phillips (1978). Gonorrhoea in women: Diagnostic, clinical and laboratory aspects. Lancet; i, 761-4

14. Thin RNT and Shaw EJ (1979). Diagnosis of gonorrhoea in women. Br.J.Vener.Dis. 55, 10-13

15. Barlow D (2007). (slide 39)

16.Turner KME, et al (2014) An early evaluation of clinical and economic costs and benefits of implementing point of care NAAT tests for chlamydia trachomatis and Neisseria gonorrhoeae in genitourinary medicine clinics in England. Sex Transm Infect; 90:104-111

17. Barlow D (2007). (Slide 32)

18. Belsey EM (1983). Diagnosis of gonorrhoea in women - a national survey. Br.J.Vener.Dis. 59, 59-62

19. Mitchell S and Barlow D (1988) unpublished data

20. Morton RD (1977) Gonorrhoea. W B Saunders 
Company Ltd, London, p 41

21.Unemo M and Ison C (2013) WHO. Laboratory diagnosis of sexually transmitted infections. Gonorrhoea. p21-54

apps.who.int/iris/bitstream/10665/85343/1/978924150 5840_eng.pdf

22. Taylor E and Phillips I (1979). Assessment of $a$ selective medium for the isolation of Neisseria gonorrhoeae. Br.J.Vener.Dis. 55, 183-185

23. Taylor E and Phillips I (1980). Assessment of transport and isolation methods for gonococci. Br.J.Vener.Dis. 56, 390-393

24. Mannion PK, Fairley CK, Fehler G et al (2016). Trends in gonorrhoea positivity by nucleic acid amplification test versus culture among Australian heterosexual men with a low prevalence of gonorrhoea, 2007-2014. Sex Transm Infect 2016; 92:625-628 doi: 10.1136/sextrans-2015052246 